\title{
SEGURANÇA DO PACIENTE NA PRÁXIS DO CUIDADO DE ENFERMAGEM: PERCEPÇÃO DE ENFERMEIROS
}

\author{
PATIENT SAFETY IN THE PRACTICE OF NURSING CARE: \\ NURSES' PERCEPTIONS
}

\section{SEGURIDAD DEL PACIENTE EN LA PRÁCTICA DE ENFERMERÍA: PERCEPCIONES DE ENFERMERAS}

\author{
Renata Terezinha da Rosa* \\ Maria Helena Gehlen ${ }^{* *}$ \\ SilOMAR ILHA ${ }^{* * *}$ \\ FABIANi WeIsS PEREIRA*** \\ Talita Cassola ${ }^{* * * * *}$ \\ DiRCE STEIN BACKes*****
}

\begin{abstract}
RESUMO
São considerados princípios que qualificam o cuidado de enfermagem e direciona a prática baseada em evidência, com ética e compromisso profissional, a realização de procedimentos seguros e adequados aos pacientes. Objetivo: Identificar a percepção dos enfermeiros sobre a segurança do paciente na práxis do cuidado de enfermagem. Método: Pesquisa descritiva, exploratória de caráter qualitativo, realizada com nove enfermeiros de um hospital de pequeno porte, localizado na região central do Rio Grande do Sul. Os dados foram coletados no período de agosto a novembro do ano de 2011 por meio de entrevista semi-estruturada e foram submetidos à análise de conteúdo. Resultados: Emergiram quatro categorias: Segurança do Paciente: visibilidade na organização do cuidado de enfermagem; Segurança do Paciente: um devir a ser construído no cuidado de enfermagem; Desorganização do ambiente e do profissional: fatores que contribuem para o erro; Diferentes posturas do enfermeiro frente a situação do erro. Conclusões: A segurança do paciente poderá ser efetivada a partir do conhecimento, compromisso e participação de todos os profissionais, sendo necessário desenvolver um movimento cultural e transformador na práxis do cuidado de enfermagem.
\end{abstract}

Palavras chave: Segurança do paciente, gerenciamento de segurança, enfermagem.

\footnotetext{
*Enfermeira, graduada pelo Centro Universitario Franciscano, Santa Maria, Rio Grande do Sul, Brasil. E-mail: renatadarosa1@ hotmail.com

${ }^{* *}$ Enfermeira, professora do curso de Enfermagem do Centro Universitário Franciscano, Santa Maria, Rio Grande do Sul, Brasil. E-mail: mahgehlen@terra.com.br

${ }^{* * *}$ Enfermeiro, aluno de Doutorado em Enfermagem, Programa de Pós-Graduação em Enfermagem (PPGEnf), Universidade Federal do Rio Grande (FURG), Rio Grande, Rio Grande do Sul, Brasil. E-mail: silo_sm@hotmail.com

${ }_{* * * *}$ Enfermeira, aluna do Mestrado em Enfermagem, Programa de Pós Graduação em Enfermagem (PPGEnf), Universidade Federal do Rio Grande (FURG), Rio Grande, Rio Grande do Sul, Brasil. E-mail: enffabiweiss@hotmail.com

${ }_{* * * * *}$ Enfermeira, graduada pelo Centro Universitário Franciscano, Santa Maria, Rio Grande do Sul, Brasil. E-mail: talita_ cassola@hotmail.com

${ }_{* * * * * *}$ Enfermeira, professora do Programa de Pós-Graduação em Enfermagem (PPGEnf), Universidade Federal do Rio Grande (FURG), Rio Grande, Rio Grande do Sul, Brasil. E-mail: backesdirce@ig.com.br
} 


\begin{abstract}
Ethical and professional commitment and the application of safe and adequate procedures to patients are considered principles guiding nursing care provision and evidence-based practice. This study aims, therefore, to identify the perceptions of nurses about patient safety in the practice of nursing care. Method: Descriptive, qualitative, exploratory study conducted with nine nurses at a small hospital located in the central region of the State of Rio Grande do Sul, Brazil. The data were collected from August to November 2011 using semistructured interviews and were subjected then to content analysis. Results: Four categories emerged: Patient Safety: visibility in the organization of nursing care; Patient Safety: transformation based on nursing care; Disorganization of working and professional environment: factors contributing to errors; Different attitudes towards errors. Conclusion: Patient safety is achieved through the knowledge, commitment and participation of all professionals contributing to create a cultural change and transformative practice of nursing care.
\end{abstract}

Key words: Patient safety, safety management, nursing.

\title{
RESUMEN
}

Son considerados principios que cualifican los cuidados de enfermería y dirigen la práctica basada en la evidencia, el compromiso ético y profesional, la realización de procedimientos seguros y adecuados para los pacientes. Objetivo: Identificar las percepciones de las enfermeras acerca de la seguridad del paciente en la práctica de los cuidados de enfermería. Método: Investigación cualitativa, descriptiva exploratoria, realizada con nueve enfermeras en un pequeño hospital, ubicado en la región central de Río Grande do Sul. Los datos fueron recolectados entre agosto y noviembre de 2011 a través de entrevista semiestructurada y fueron sometidos a análisis de contenido. Resultados: Cuatro categorías emergieron: Seguridad del Paciente: visibilidad en la organización de los cuidados de enfermería; Seguridad del Paciente: un devenir que se construye en los cuidados de enfermería; Desorganización del medio ambiente y profesional: factores que contribuyen al error; Diferentes posturas de la enfermera en el estado de error. Conclusiones: La seguridad del paciente se puede hacer a partir del conocimiento, el compromiso y la participación de todos los profesionales, siendo necesario desarrollar un movimiento cultural y praxis transformadora de la atención de enfermería.

Palabras clave: Seguridad del paciente, administración de la seguridad, enfermería.

Fecha recepción: 13/09/14 Fecha aceptación: 04/11/15

\section{INTRODUÇÃO}

Realizar os procedimentos seguros e adequados às necessidades dos pacientes hospitalizados, objetivando alcançar os melhores resultados, são princípios que qualificam o cuidado de enfermagem e direcionam a prática baseada em evidência com ética e compromisso profissional. Nesse aspecto, a segurança dos pacientes tem merecido a atenção da Organização Mundial da Saúde (OMS), uma vez que os erros representam um grave prejuízo tanto à saúde de indivíduos quanto a custos financeiros para o país (1).

No Reino Unido e na Irlanda do Norte, o prolongamento do tempo em que o paciente permanece hospitalizado, devido a erros, custa cerca de dois bilhões de libras ao ano, e o gasto anual do Sistema Nacional de Saúde com questões associadas a eventos adversos é de 400 milhões de libras. Nos EUA, estima-se um gasto de 17 a 29 bilhões de dólares por ano (1). No final da década de 1990 e início do ano 2000, o Brasil apresentou-se como um ambiente propício para a incorporação 
de ações e assistência à saúde voltadas à segurança do paciente (2).

Dentre as ações para a segurança do paciente, encontra-se a criação, no ano de 1999 da Agência Nacional de Vigilância Sanitária (ANVISA), pelo Ministério da Saúde no intuito de garantir a segurança sanitária dos produtos e serviços. Outra ação foi à criação de comitês multidisciplinares denominados Comitês de Qualidade, Comitê de Farmácia e Terapêutica ou Comitê de Segurança do Paciente. Em Concepción, no Chile, a Organização Pan-Americana da Saúde criou, no ano de 2005, a Rede Internacional de Enfermagem e Segurança do Paciente e, em 2008, foi criada a Rede Brasileira de Enfermagem e Segurança do Paciente (REBRAENSP), que objetiva fortalecer a assistência de enfermagem segura e de qualidade (2).

A segurança do paciente no que concerne o cuidado em enfermagem, caracteriza-se pela redução do risco de danos desnecessários associados à assistência em saúde. Entende-se que a situação de erro poderá ocorrer no cotidiano do cuidado de enfermagem seja por imperícias, negligências, imprudências, omissões e que esses erros, muitas vezes, independem da excelência da qualificação do profissional da saúde. Todavia a segurança do paciente é uma responsabilidade legal da enfermagem em garantia a atenção integral do cuidado (3).

Alguns estudos sobre a segurança do paciente vêm sendo realizados em diferentes contextos (4-7). No entanto há uma lacuna do conhecimento acerca da percepção dos enfermeiros sobre a temática, reforçando a importância desse estudo no que concerne à construção de um conhecimento a ser agregado ao dos já existentes acerca da temática. É importante compreender a percepção dos profissionais de saúde, em especial dos enfermeiros, que normalmente são os responsáveis pela sistematização dos cuidados aos pacientes nos diferentes cenários de atuação, referente à segurança do paciente, justificando a relevância e necessidade desse estudo.

\section{Problema}

Identificar e intervir nas possíveis situações plausíveis de gerar erros é dever do enfermeiro no seu exercício profissional. A abordagem tradicional utilizada em alguns locais é direcionada a culpar unicamente o profissional que errou em algum momento, durante sua atividade de cuidados ao paciente. Porém, ao longo da última década, tem-se reconhecido que essa abordagem subestima o fato de que alguns erros são causados por profissionais comprometidos e qualificados (3).

Vale ressaltar que o cuidado se desenvolve em um cenário complexo e dinâmico com situações passíveis de predispor o erro. Desta forma, garantir a segurança do paciente é um compromisso que visibiliza a organização e liderança no cuidado de enfermagem. Estudos sobre a segurança do paciente, bem como sobre participação do enfermeiro na implantação de estratégias para a melhoria da qualidade e da segurança da assistência de enfermagem são ainda recentes e necessários (8). Frente ao exposto questiona-se: qual a percepção dos enfermeiros sobre a segurança do paciente no cenário hospitalar?

Nas últimas décadas, a preocupação com a segurança no cuidado prestado ao paciente tem se tornado um dos assuntos prioritários na área da saúde, refletindo na busca e desenvolvimento de evidências científicas. No final da década de 1990, a temática da segurança do paciente repercutiu fortemente nos Estados Unidos da América (EUA) e posteriormente, a partir da publicação do relatório "To err is human" pelo Institute of Medicine dos EUA, expandiu-se a outros países como o Brasil, Peru, Argentina e Colômbia (9).

No Brasil a preocupação com a temática da segurança do paciente torna-se relevante ao considerar a complexidade do Sistema Único de Saúde (SUS), o qual é financiado com recursos públicos, com diversos fatores considerados agravantes, tanto pela questão estrutural, política, econômica e cultural, as quais devem ser superadas para garantir a 
qualidade nos serviços. É possível visualizar, com frequência, os meios de comunicação abordando as más condições de trabalho, recursos escassos, superlotações e longas esperar nos serviços de emergência de hospitais públicos, potencializando, de certa forma, os erros dos profissionais e as falhas na assistência ao paciente (4).

Pesquisas estão demonstrando que a satisfação do profissional com o trabalho é um fator que está diretamente relacionado com a qualidade da assistência prestada. A insatisfação do profissional enfermeiro pode estar relacionada, dentre outros aspectos, ao acúmulo de atividades e às escassas perspectivas de obter novos conhecimentos, prejudicando a qualidade do seu desempenho junto com o paciente. Nesse contexto, o conhecimento pode ser pensado como uma ferramenta importante para os profissionais de saúde garantirem cuidados seguros e de alta qualidade aos pacientes (10).

Assim, emerge a necessidade do conhecimento do enfermeiro acerca da segurança do paciente, com vistas a garantir o cuidado efetivo de forma segura e identificar as possíveis falhas, objetivando solucioná-las. Alguns estudos tem sido realizados com vistas a conhecer a percepção dos profissionais de enfermagem acerca da temática em pauta. Estudo realizado em um Hospital Universitário do Sul do Brasil demonstrou que os membros da equipe de enfermagem convergem em suas percepções acerca dos riscos para a segurança do paciente, pois identificaram fatores de risco, que não possuem relação com o conceito da OMS (11).

Estudo realizado com 20 enfermeiros no estado de São Paulo (SP) evidenciou que os profissionais reconhecem como o principal objetivo da segurança do paciente, a assistência livre de danos (12). A percepção dos enfermeiros está ancorada de acordo com o Código de Ética dos profissionais de enfermagem que, no artigo 16, faz referência à responsabilidade de garantir, ao paciente, assistência de enfermagem segura e livre de da- nos de qualquer natureza, seja por imperícia, negligência ou imprudência (13).

Entretanto, até o momento, ainda são insipientes os estudos que versem acerca da segurança do paciente na perspectiva dos profissionais da saúde, necessitando esse tema ser explorado e em contextos diversos. Assim, apresenta-se indispensável conhecer a percepção dos profissionais, especialmente dos enfermeiros que são os responsáveis pela liderança da equipe de enfermagem e, portanto, precisam estar preparados para atuar com as situações que se apresentam no cotidiano de cuidados ao paciente (12).

Objetivo: Identificar a percepção dos enfermeiros sobre a segurança do paciente na práxis do cuidado de enfermagem.

\section{MATERIAL E MÉTODO}

Trata-se de uma pesquisa descritiva, exploratória de caráter qualitativo (14), realizada com enfermeiros de um hospital de pequeno porte vinculado ao Sistema Único de Saúde (SUS), localizado na região central do Rio Grande do Sul (RS), Brasil. O hospital em questão possuía, no momento em que se desenvolveu esse estudo, as unidades de clínica médica e cirúrgica, bloco cirúrgico e Centro de Materiais de Esterilização (CME), Unidade de Pediatria e Maternidade. Contava com a Comissão de Controle de Infecção Hospitalar (CCIH) e com a gerência de Enfermagem. Para esse estudo, consideraram-se todos os setores do hospital em questão. Nesse cenário, atuavam profissionais de saúde de diversas áreas, entre eles, nove enfermeiros.

Como critérios de inclusão estabeleceram-se: ser enfermeiro e atuar no cenário escolhido para essa pesquisa. E, como critérios de exclusão os profissionais que estivessem de férias ou afastados por algum problema de saúde. Atenderam aos critérios de inclusão formando o Corpus desse estudo, os nove 
enfermeiros da instituição.

Optou-se por não diferenciar os enfermeiros assistenciais dos gerenciais, por compreender que a segurança do paciente é uma temática relevante e que, portanto, precisa permear os diversos campos nos quais essa realidade se insere. Dessa forma, compreende-se a importância de se identificar a percepção, tanto dos enfermeiros que estão diretamente envolvem no cuidado, quanto nos que gerenciam esse processo.

Os dados foram coletados entre os meses de agosto a novembro do ano de 2011, por meio da entrevista semi-estruturada contemplando às questões norteadoras: Para você, o que significa segurança do paciente? Se alguém cometer um erro, o que isso significa? Qual a sua conduta ao saber que um funcionário cometeu um erro? Descreva as principais situações que poderão influenciar para que ocorra o erro no serviço de enfermagem. As entrevistas foram realizadas individualmente com cada profissional em uma sala do próprio hospital mediante agendamento prévio.

Os dados foram analisados, interpretados e categorizados por meio da análise de conteúdo. Essa técnica consiste em descobrir os núcleos de sentido que compõem uma comunicação, cuja presença ou frequência acrescentem perspectivas significativas ao objeto de estudo em questão. A noção da temática está associada a uma afirmação que diz respeito a um determinado assunto, podendo ser apresentada por uma palavra, frase ou idéia (15).

Desse modo, a operacionalização do processo de análise seguiu as três etapas do método. Na primeira etapa, denominada de pré-análise, se buscou fazer uma leitura exaustiva dos dados, seguida da organização do material e da formulação de hipóteses. Na sequência, foi realizada a exploração do material e se buscou codificar os dados brutos. Na terceira e última fase, os dados foram interpretados e delimitados em eixos temáticos, de acordo com os significados atribuídos (15).
Foram considerados os preceitos éticos e legais, vigentes na época deste estudo, que envolvem a pesquisa com seres humanos, conforme resolução 196/96 do Ministério da Saúde (16). Foi distribuído anteriormente o Termo de Consentimento Livre e Esclarecido (TCLE) para os participantes da pesquisa, sendo este em duas vias, ficando uma com o participante e outra com os pesquisadores. Manteve-se o anonimato dos depoentes e os mesmos foram identificados pela palavra "Entrevistado", seguida de um algarismo numérico conforme ordem de entrevista (Entrevistado 1, Entrevistado 2... Entrevistado 9). O projeto foi aprovado pelo Comitê de Ética em Pesquisa (CEP) do Centro Universitário Franciscano, sob o no 196.2011.2.

\section{RESULTADOS}

Dos enfermeiros participantes desse estudo três atuavam na unidade de clínica médica, um na unidade de clínica cirúrgica, um no bloco cirúrgico e CME, um na unidade de pediatria, um na maternidade, um no $\mathrm{CCIH}$ e um na gerência de Enfermagem.

Dos dados organizados e analisados, emergiram quatro categorias: Segurança do Paciente: visibilidade na organização do cuidado de enfermagem; Segurança do Paciente: um devir a ser construído no cuidado de enfermagem; Desorganização do ambiente e do profissional: fatores que contribuem para o erro; Diferentes posturas do enfermeiro frente à situação do erro.

\section{Segurança do Paciente: visibilidade na or- ganização do cuidado de enfermagem}

Os enfermeiros relacionaram a segurança com a permanência do paciente no hospital e à organização do cuidado de enfermagem voltado para a atenção integral e à promoção da humanização. Por conseguinte os relatos: 
Segurança do paciente inclui todas as situações que acontece dentro do hospital, desde a entrada dele na recepção, até a alta. Todos os passos que acontecem aqui dentro [....] tudo que envolve procedimento e rotina é segurança do paciente pra mim. (Entrevistado 2)

Segurança do paciente são todos os critérios que a gente tem pra que o paciente não corra algum risco dentro da instituição, tudo que foi desde a parte de tranqüilizar o paciente até um erro na medicação, tudo que envolva a confiança para o paciente dentro da instituição. (Entrevistado 4) Segurança do paciente é como tu vai cuidar ele (paciente), é como tu vai tratar, engloba o todo, o teu tratamento perante o paciente, como tu vai lidar com o familiar. (Entrevistado 3)

Nos relatos é possível observar que os enfermeiros mesmo não possuindo um conhecimento concreto do que se trata segurança do paciente, possuem uma percepção ampliada a respeito da temática, onde relacionam com todo o cuidado o que poderá proporcionar o bem estar do paciente. As percepções dos enfermeiros também se remeteram à importância das discussões sob o fazer profissional no cotidiano do cuidado de enfermagem.

\section{Segurança do Paciente: um devir a ser cons- truído no cuidado da enfermagem}

A segurança do paciente não é uma realidade ainda problematizada como responsabilidade individual e coletiva de todos os profissionais. Todavia poderá ser construída no exercício profissional, pois há possibilidades de transformação das percepções dos sujeitos para o vivido concreto na práxis cotidiana do cuidado de enfermagem. A percepção conceitual de segurança dos sujeitos foi contextualizada como um déficit na formação profissional do enfermeiro. Relacionando-a com a efetividade da segurança do paciente no cotidiano, Conforme os relatos a seguir:

Segurança do paciente? Na minha formação a gente não enfatizou, isso é uma coisa bem nova pra gente no Brasil. Eu acredito que lá fora já éa muito tempo trabalhado. (Entrevistado 5)

[...] eu nunca havia ouvido falar em segurança do paciente, acho que é um tema novo, não é? (Entrevistado 1)

[...] não me lembro de ter estudado a respeito disso na graduação, mas já ouvi falar algo a respeito. (Entrevistado 9)

Evidencia-se o desconhecimento dos profissionais quanto a Segurança do Paciente. Isso ocorre, segundo os profissionais, em decorrência dessa temática não ter sido abordada no seu processo de formação.

\section{Desorganização do ambiente e do profissio- nal: fatores que contribuem para o erro}

Ao questionar os profissionais entrevistados quanto aos motivos que podem propiciar o erro, os mesmos, referiram que a desorganização e a superlotação do setor de saúde, barulho, stress profissional entre outros fatores podem ser agravantes para um mau funcionamento do serviço, uma vez que dificultam a concentração dos profissionais. Conforme as falas a seguir:

O principal item que leva a um erro é a desorganização, um ambiente desorganizado, as pessoas desorganizadas irá facilitar o erro. (Entrevistado 4)

Eu acredito que quando tem uma super lotação no setor, o stress do funcionário com a carga horária, com os familiares, o barulho, muitas pessoas, muito visitantes, isso dificulta a concentração do funcionário. (Entrevistado 1)

Nos relatos torna-se evidente que a desorganização do ambiente de trabalho, bem como a superlotação, que hoje é uma realidade em alguns hospitais brasileiros, dificulta a concentração do profissional no seu fazer, fato que segundo os enfermeiros, sujeitos desse estudo, poderá influenciar para a ocorrência do erro. Um enfermeiro sugeriu ainda, 
que a excessiva carga horária de trabalho na área da enfermagem, associado a outros fatores, dificulta a concentração do profissional, podendo propiciar o erro.

\section{Diferentes posturas do enfermeiro frente à situação do erro}

Observa-se que a conduta dos enfermeiros quando ocorre uma iatrogenia no cuidado ao paciente, são mencionadas de duas maneiras distintas. Pode ocorrer de forma vertical, ou seja, de cima para baixo, onde o enfermeiro pune, reprime ou de forma horizontal, por meio da liderança, onde os profissionais procuram utilizar a educação como ferramenta de mudança em prol da terapêutica adequada as necessidades do contexto. A seguir os relatos:

[...] não adianta punir um erro no ambiente de trabalho e sim ver por quem e o porquê, pra tentar corrigir, seja com treinamento, seja chamando toda a equipe [...] errar é humano, não adianta tu punir e expor o funcionário [...]. (Entrevistado 2)

O primeiro passo é entender o que levou aquela pessoa a errar, o que aconteceu que ela apresentou essa falha, tem que conversar, eu não posso amedrontar, não posso coagir [...]. (Entrevistado 4)

Todo mundo é passivo de erros né, cabe a coordenação avaliar os erros e tomar as providencias em cima de cada erro. (Entrevistado 8)

Primeiro tem que saber o porquê do erro, não importa se foi grave se foi leve tem que dar advertência, o profissional tem que ser advertido [...]. (Entrevistado 3)

Primeiro lugar é chamar e conversar o porquê do erro, se não sabia, se foi falta de atenção e dar uma advertência [...]. (Entrevistado 9)

Salienta-se que os sujeitos consideraram que errar é humano e que a responsabilidade cabe ao responsável que está coordenando a equipe. No entanto, a conduta diverge entre os profissionais, permeando entre advertên- cia e compreensão do profissional como ser humano cabível de errar. Também se inferiu que a segurança do paciente deve ser medida e pesada frente ao erro já cometido, o que pode ser analisado como uma lacuna na prevenção de sua ocorrência.

\section{DISCUSSÃO E CONCLUSÃO}

Os enfermeiros participantes desse estudo relacionaram a segurança do paciente com a organização do cuidado de enfermagem, a promoção da humanização e todo o cuidado que poderá proporcionar o bem estar do paciente. Foi possível identificar, ainda, um déficit na formação profissional do enfermeiro com relação à temática da segurança do paciente, pois os mesmos referiram que essa temática não lhes foi abordada no processo de formação. Estudo realizado com profissionais de enfermagem identificou que a maioria dos participantes não conheciam os meios adequados para encaminhar as questões relacionadas à segurança do paciente (17).

O desconhecimento pode estar atrelado ao fato de que as discussões e estudos acerca dessa temática são relativamente recentes, considerando-se os aspectos históricos da saúde. Esse fato impõe o desafio na formação dos profissionais de saúde, especialmente a do Enfermeiro, que é o profissional responsável pelo sistematização do cuidado realizado diretamente aos pacientes em diferentes cenários (18). Dessa forma evidencia-se que temática segurança do paciente deve estar inserida em todo o currículo de formação desse profissional, com atividades que propiciem ao aluno e professor experienciar práticas que repercutam em uma atuação segura (19).

Faz-se necessária uma (re)organização no contexto de formação dos futuros enfermeiros, com incentivo à pesquisa e à prática baseada em evidências atrelada à temática da segurança do paciente. Além do mais as ins- 
tituições de saúde devem compreender que a segurança do paciente é a segurança do próprio profissional (20), incentivando a educação permanente dos profissionais e sensibilizando-os para essa temática.

Alguns pesquisadores têm investigado a efetivação da segurança em instituições de saúde enquanto processo cultural, de modo a ser reconhecida como importante estratégia para a melhoria do cuidado, suprindo a lacuna existente no aspecto da segurança do paciente (21). Investir em uma cultura de segurança nas instituições de saúde poderá reunir valores tanto individuais como coletivo, atitudes, percepções, competências e padrões de comportamentos, que determinam o compromisso, o estilo e capacidade da organização de saúde, refletido pelo gerenciamento da segurança do paciente (22).

Nesse estudo pode-se perceber a divergência em relação à conduta dos profissionais em relação ao erro no cuidado ao paciente. Alguns referem ações punitivas, enquanto outros, a educação como ferramenta de mudança com intuito do alcance da terapêutica adequada. Estudo realizado com objetivo de avaliar a percepção do clima de segurança dos profissionais de enfermagem atuantes nas clínicas médicas e cirúrgicas de um hospital de ensino identificou que ainda predomina entre os profissionais a percepção de que falhas na segurança do paciente resultam apenas em punições para o profissional (17).

Estudo realizado em Campos do Jordão com 52 enfermeiros identificou que quando na ocorrência de um erro por parte do profissional, houve o predomínio de $49 \%$ de advertência verbal e 33\% de escrita. Foram relatadas também como medidas punitivas, a suspensão (11\%), demissão (5\%) e assédio moral (2\%) (21). Cabe salientar que o erro é comumente associados apenas a falha do profissional que o cometeu. No entanto, devem ser consideradas as condições de trabalho, os aspectos estruturais e a complexidade das atividades desenvolvidas como fatores que podem desencadear ou predispor o mesmo (8).

Faz-se necessário, nesse contexto, a comunicação efetiva e de qualidade dentro dos membros da equipe de saúde, pois dessa forma torna-se possível a identificação de oportunidades que venham a contribuir para uma melhor atenção aos pacientes (22). É necessário compreender os possíveis motivos que induziram o profissional ao erro, procurar estratégias capazes de desmistificar a visão de um cenário de saúde marcado pelo descaso no atendimento aos pacientes (23). Tais estratégias merecem ser discutidas e abordadas desde o processo formativo de profissionais da saúde, na possibilidade de tornar-se uma cultura com reflexos na prática do cuidado.

Perante tais iniciativas, torna-se necessário, a compreensão junto à equipe de saúde, da importância de potencializar mudanças, assimilando a cultura de segurança como um processo de socialização que permite o (re) pensar na assistência à saúde, tanto na perspectiva de educação permanente aos profissionais atuantes no sistema, como transformar tais cenários de ensino-aprendizagem para futuros profissionais. No entanto, compreende-se que criar/transformar uma cultura pode ser um processo longo, porém necessário e possível, no que concerne a qualidade do cuidado ao paciente.

Os profissionais desse estudo referem à desorganização e a superlotação do setor de saúde; o barulho; o stress profissional e a excessiva carga de trabalho como fatores que podem influenciar para o mau funcionamento do serviço e dificultar a concentração dos profissionais, propiciando o erro. Esse resultado se assemelha a um estudo realizado com profissionais de enfermagem do estado de São Paulo, o qual identificou que de 203 profissionais $167(82 \%)$ acreditam que a carga excessiva de trabalho prejudica o seu desempenho profissional; 155 (78\%) concordam que são menos eficientes quando estão cansados e 158 (78\%) referem que possuem 
maior probabilidade de cometer erros em situações tensas e hostis (17).

Estudo que objetivou analisar a carga de trabalho da equipe de enfermagem e sua potencial associação com a segurança do paciente nas unidades de internação clínica e cirúrgica de um hospital do Sul do Brasil, evidenciou associação entre o aumento do número de pacientes aos cuidados da equipe de enfermagem com a elevação de quedas do leito, infecções associadas ao cateter venoso central, absenteísmo e rotatividade de profissionais (4).

Assim, evidencia-se que a enfermagem, como profissão que assume papéis de liderança nas instituições de saúde (18), deve preocupar-se com a segurança e qualidade do cuidado aos pacientes. Nesta perspectiva, os resultados oriundos deste estudo abrem caminho para o (re)pensar em projetos/estratégias futuras a serem desenvolvidas com vistas a explorar as lacunas existente tanto no processo formativo de profissionais como a realização de uma educação permanente efetiva, que consiga dar conta de evitar e remediar riscos e agravos relacionados a segurança de pacientes.

Considera-se satisfatória a realização desse estudo, pois foi possível identificar a percepção dos enfermeiros sobre a segurança do paciente na práxis do cuidado de enfermagem. Pode-se perceber que, alguns profissionais, consideram que realizar os cuidados corretos e seguros, na busca de alcançar os melhores resultados possíveis, são princípios que fundamentam a qualidade da assistência prestada ao paciente.

Esse estudo possui limitações comuns a quaisquer estudos qualitativos, sobretudo há escassez de estudos acerca da percepção dos enfermeiros sobre a temática segurança do paciente no Brasil e em muitos países, portanto divulgar essa pesquisa no meio científico torna-se relevante, pois por meio dela pode-se instigar novos pesquisadores acerca de uma temática de extrema relevância. Como potencialidades, o estudo possibilitou identificar que a segurança do paciente tem que ser revista no seu principal objetivo e o que ela representa no serviço de enfermagem, no cotidiano dos pacientes que tem o direito de receber cuidados dignos, com qualidade em suas necessidades individuais e integrais.

A segurança do paciente é mais do que encontrar culpados à punição, o que tem que ser investigado são as lacunas do serviço prestado ao paciente e com isso traçar estratégias para prevenir as incidências e reincidências do erro, garantindo a redução dos danos causados por erros na assistência. Salienta-se que a segurança do paciente será efetiva a partir do conhecimento, compromisso e participação de todos os profissionais em prol do ser humano de maneira digna e respeitosa, sendo necessário desenvolver um movimento cultural e transformador na práxis do cuidado de enfermagem.

Acredita-se que o assunto abordado como tema do estudo possa gerar reflexões/discussões nas opiniões formadas pela sociedade, e profissionais de saúde e colaborar com a ciência da enfermagem/saúde para um maior conhecimento acerca da segurança do paciente. Espera-se que este estudo contribua para a comunidade científica, instigando outros pesquisadores da área da enfermagem/saúde a realizarem novas pesquisas com vistas a identificarem as percepções dos profissionais de enfermagem/saúde acerca dessa temática, potencializando possíveis intervenções de ensino aprendizagem tanto no processo de formação por meio das discussões nas disciplinas, quanto no serviço por meio da educação permanente.

Compreendendo a importância da temática sugere-se a realização de mais estudos que possam agregar conhecimentos novos aos estudos já existentes, para que em um futuro próximo, a efetivação da segurança do paciente seja uma realidade instituída em todos os cenários de ensino e atuação dos profissionais de enfermagem/saúde. 


\section{REFERÊNCIAS}

1. Mendes W, Shcolnik W. Segurança na saúde: Jornal O Globo. 5 de Março de 2011: 2.

2. Cassiani SHDB. Nursing and the Research on Patient Safety. Acta Paul Enferm. 2010; 23(6): Vii-Viii.

3. Wachter RM. Compreendendo a segurança do paciente; Tradução: Laura Souza Berquó. Porto Alegre: Editora Artmed, 2010.

4. Magalhães AMM, Dall'agnol CM, Marck PB. Nursing workload and patient safety - a mixed method study with an ecological restorative approach. Rev Lat Am Enfermagem. 2013; 21(esp): 146-154.

5. Raduenz AC, Hoffmann P, Radunz V, Dal Sasso GTM, Maliska ICA, Marck PB. Nursing Care and Patient Safety: Visualizing Medication Organization, Storage and Distribution with Photographic Research Methods. Rev Lat Am Enfermagem. 2010; 18(6): 1045-54.

6. Capucho HC, Arnas ER, Cassiani SHBD. Segurança do paciente: comparação entre notificações voluntárias manuscritas e informatizadas sobre incidentes em saúde. Rev Gaúcha Enferm. 2013; 34(1): 164172.

7. Amorim FDB, Flores PVP, Bosco PS, Menezes AHB, Alóchio KV. The scheduling of medicines guided in patient safety: a warning for nursing practice. Rev enferm UFPE on line [Internet]. 2014 [citado 05 ago 2014]; 8(1): 224-8. Disponível em: file://C:/Users/Andre/Downloads/564451626-1-PB.pdf

8. Oliveira RM, Leitão IMTA, Silva LMS, Figueiredo SV, Sampaio RL, Gondim MM. Strategies for promoting patient safety: from the identification of the risks to the evidence-based practices. Esc. Anna Nery. 2014; 18(1): 122-9.

9. Bates DW. Preventing medication errors: a summary. Am J Health Syst Pharm. 2007; 64(14Suppl9): S3-9; quiz S24-6.

10. Reis AM, Marques TC, Opitz SP, Silva AE, Gimenes FR, Teixeira TC, et al. Errors in medicine administration - profile of medicines: knowing and preventing. Acta Paul Enferm. 2010; 23(2): 181-6.

11. Souza FT, Garcia MC, Rangel PPS, Rocha PK. Percepção da enfermagem sobre os fatores de risco que envolvem a segurança do paciente pediátrico. Rev Enferm UFSM [Internet]. 2014 [citado 03 nov 2015]; 4 (1): 152-62. Disponível em: http://cascavel.ufsm.br/revistas/ojs-2.2.2/index.php/ reufsm/article/view/8781/pdf

12. Dias JD, Mekaro KS, Tibes CMS, Zem-Mascarenhas SH. The nurses' understanding about patient safety and medication errors. Rev Min Enferm [Internet]. 2014 [citado 03 nov 2015]; 18(4): 874-80. Available from: http://www.reme.org.br/ artigo/detalhes/969

13. Conselho Federal de Enfermagem. Código de Ética dos Profissionais de Enfermagem. Resolução COFEN No160/93 de 12 de maio de 1993. Rio de Janeiro: COFEN; 1993.

14. Cansonieri AM. Metodologia da pesquisa qualitativa na saúde. Rio de Janeiro: Vozes; 2010.

15. Bardin L. Análise de conteúdo. 4a Ed. Lisboa: Edições, 2009.

16. Ministério da Saúde (BR). Conselho Nacional de Saúde. Diretrizes e normas regulamentadoras de pesquisa envolvendo seres humanos. Resolução n. 196, de 10 de outubro de 1996. Brasília; 1996.

17. Rigobollo MCG, Carvalho REFL, Cassiani SHB, Galon T, Capucho HC, Deus NN. The climate of patient safety: perception of nursing professionals. Acta Paul Enferm. 2012; 25(5): 728-35.

18. Waldow VR. Bases e princípios do conhecimento e da arte da enfermagem. Petrópolis (RJ): Vozes; 2008.

19. Urbanetto JS, Gerhardt LM. Segurança 
do paciente na tríade assistência ensino pesquisa [Editorial]. Rev Gaúcha Enferm. 2013; 34(3): 8-9.

20. Ques AAM, Montoro CH, González MG. Strengths and Threats Regarding the Patient's Safety: Nursing Professionals' Opinion. Rev Lat Am Enfermagem. 2010; 18(3): 339-45.

21. Claro CM, Krocockz DVC, Toffolleto MC, Padilha KG. Adverse events at the Intensive Care Unit: nurses' perception about the culture of no-punishment. Rev
Esc Enferm USP. 2011; 45(1): 167-72.

22. Ramírez OG, Gutiérrez WA, Vega LG, Salamanga JG, Galeano EM, Gámez AS. Cultura de seguridad del paciente por personal de enfermería em Bogotá, Colombia. Cienc enferm. 2011; XVII(3): 97111.

23. Pedreira MLG, Harada MJCS. Aprendendo com os erros. En: Harada MJCS, Pedreira MAS, Pereira SR, Editors. O erro humano e a segurança do paciente. São Paulo: Atheneu; 2006. P. 175-84. 